\title{
The Amino Acid Distribution in Laboratory Analogs of Extraterrestrial Organic Matter: A Comparison to CM Chondrites
}

\author{
P. Modica ${ }^{1,2}$ (1D) Z. Martins ${ }^{3,4}$ (D) C. Meinert ${ }^{5}$, B. Zanda ${ }^{6}$, and L. L. S. d'Hendecourt ${ }^{1,7}$ \\ ${ }^{1}$ CNRS-Université Paris XI, Institut d'Astrophysique Spatiale, "Astrochimie et Origines," F-91405 Orsay Cedex, France \\ ${ }^{2}$ Currently at: Laboratoire de Physique et Chimie de l'Environnement et de l'Espace (LPC2E), CNRS/Université d'Orléans, F-45071 Orléans, France \\ ${ }^{3}$ Department of Earth Science and Engineering, Imperial College London, South Kensington Campus, London SW7 2AZ, UK \\ ${ }^{4}$ Currently at: CQFM-IN and IBB, Departamento de Engenharia Química, Instituto Superior Técnico, \\ Universidade de Lisboa, Avenida Rovisco Pais 1, 1049-001 Lisboa, Portugal \\ ${ }^{5}$ Université Côte d'Azur, Institut de Chimie de Nice, UMR 7272 CNRS, 28 Avenue Valrose, F-06108 Nice, France \\ ${ }^{6}$ Muséum d'Histoire Naturelle, CNRS, 61 rue Buffon, F-75005, Paris, France \\ ${ }^{7}$ Currently at: Equipe ASTRO, Laboratoire de Physique des Interactions Ioniques et Moléculaires, UMR CNRS 7345, Centre de Saint Jérôme-case 252, \\ Université d'Aix-Marseille, F-13397 Marseille, France \\ Received 2017 October 9; revised 2018 July 31; accepted 2018 August 3; published 2018 September 19
}

\begin{abstract}
Laboratory experiments that simulate the photo- and thermo-chemistry of extraterrestrial ices always lead to the formation of semi-refractory organic residues. These residues can be considered as laboratory analogs for the primitive organic matter incorporated into comets and asteroids. Many specific organic molecules have been detected in them. Here we focus on amino acids because of their possible relevance to further prebiotic chemistry on Earth as well as in other solar system bodies. We compare the amino acid content and distribution measured in organic residues produced in our photochemical experiments to those observed in various CM chondrites presenting an increasing degree of aqueous alteration, a process that is thought to impact amino acid chemistry. We find that the amino acid profile of our residues shows similarities with that of the least aqueously altered CM chondrites. In particular, the $\beta$-alanine to glycine ratio is comparable to the one measured in the Paris meteorite, a minimally altered CM chondrite, and matches the trend followed by other CM chondrites with different degrees of aqueous alteration. Additionally, the relative abundances of $\alpha-, \beta$-, and $\gamma$-amino acids in one of our residues are similar to those of the least altered CM chondrites. These results support the idea of a general formation process for amino acids from photo- and thermo-processing of icy grains as an important source for the inventory of amino acids in the early solar system.
\end{abstract}

Key words: meteorites, meteors, meteoroids - protoplanetary disks - stars: protostars - ultraviolet: general

\section{Introduction}

The formation mechanisms and the evolution of organic matter in the solar system are still being debated (Aponte et al. 2017). It is indeed uncertain if the organic material, or at least part of it, synthetized in the parent molecular cloud, survived throughout the protosolar disk phase and was later incorporated in the planetesimals that eventually formed comets and asteroids (Messenger 2000; d'Hendecourt 2011; Bertaux \& Lallement 2017).

Among some of the remnants of comets and asteroids of the early solar system are carbonaceous chondrites. They are a class of primitive and relatively undifferentiated meteorites originating from fragments of asteroids, whose chemical composition may reflect that of their parent bodies. Carbonaceous chondrites are subdivided into the major six groups (CI, CM, CV, CO, CR, and $\mathrm{CK}$, where the $\mathrm{C}$ stands for carbonaceous and the second letter is usually the first letter of the specimen type of that group), two unusual groups that have been affected by impact processes $(\mathrm{CH}$ and CB), and many ungrouped members (Choe et al. 2010 and references therein). In particular, CM meteorites (the letter $\mathrm{M}$ stands for Mighei) are characterized by the presence of a large amount of organic materials, both soluble and insoluble, within a fine-grained $(<1 \mu \mathrm{m})$ matrix of phyllosilicates, oxides, sulfides, and carbonates (King et al. 2017).

Although carbonaceous chondrites include the most primitive known meteorites, they present different degrees of processing such as aqueous alteration by liquid water. This alteration is due to the melting of ices within the parent bodies, most probably after the heating from the decay of radioactive ${ }^{26} \mathrm{Al}$ (Grimm \& McSween 1989), and is thought to have affected the chemical content, both organic and inorganic, of these meteorites (Browning et al. 1996; Palmer \& Lauretta 2011; Vinogradoff et al. 2017).

$\mathrm{CM}$ chondrites present large variations in the degree of aqueous alteration (McSween 1979; Tomeoka \& Buseck 1985; Zolensky \& McSween 1988; Brearley 2006). In order to take into account this variability, a classification scheme was proposed by Rubin et al. (2007). It consists of a CM numerical alteration sequence based on mineralogical, petrological, and textural characteristics that correlate with increasing aqueous alteration. This scheme, more comprehensive than previous ones (e.g., McSween 1979; Browning et al. 1996), comprises different petrological subtypes. At the time of this proposition, considering only the known $\mathrm{CM}$ meteorites, the petrological subtypes ranged from 2.6-2.0 for the least to the most altered ones, respectively. Progressively, additional petrological subtypes were included for the new and least aqueously altered $\mathrm{CM}$ chondrites. One of them is the Paris meteorite, suggested to be a CM2.7 or 2.8 on this scale (Blanchard et al. 2011; Hewins et al. 2014; Marrocchi et al. 2014). However, only a very limited number of $\mathrm{CM}$ chondrites have been classified using this scheme. Recently, Alexander et al. (2013) applied the classification schemes of Browning et al. (1996) and Rubin et al. (2007) to $54 \mathrm{CM}$ meteorites by determining their bulk $\mathrm{H}$, $\mathrm{C}$, and $\mathrm{N}$ elemental abundances and isotopic compositions, 
significantly expanding the list of CM chondrites classified using petrologic subtypes.

Carbonaceous chondrites, and particularly CM meteorites, are known to contain amino acids (Cronin et al. 1988; Cronin \& Chang 1993; Martins \& Sephton 2009; Martins 2011; Burton et al. 2012). These molecules are thought to be affected by hydrothermal alteration within the asteroidal parent bodies (Elsila et al. 2016). On the one hand, the presence of liquid water could have contributed to their synthesis. The main invoked formation pathways are the Strecker reaction for $\alpha$ amino acids (Peltzer \& Bada 1978; Lerner \& Cooper 2005), Michael addition for $\beta$-amino acids (Cronin \& Chang 1993), and hydrolysis of lactams for $\gamma$ - and $\delta$-amino acids (Cronin et al. 1995). On the other hand, further hydrothermal alteration would have decomposed these molecules (Botta et al. 2007; Herd et al. 2011), favoring some groups of amino acids isomers over others (e.g., $\alpha$ - versus $\beta$ - versus $\gamma$ - amino acids) (Cooper $\&$ Cronin 1995), and influencing their distribution according to the degree of the experienced aqueous processing. From the emerging trend of this distribution, the relative abundance of $\beta$ alanine ( $\beta$-alanine/glycine) was proposed as an indicator for the degree of the experienced aqueous alteration (Glavin et al. 2006). This observation is confirmed by the study of the amino acid content, molecular distributions, and abundances of the Paris meteorite (Martins et al. 2015). In this latter work the amino acid distribution of Paris is compared to that of several $\mathrm{CM}$ chondrites exhibiting different degrees of aqueous alteration. Considering a set of 4-carbon amino acids present in the examined meteorites, the relative abundance of $\alpha$-amino acids is higher than $\beta$ - and $\gamma$-amino acids for the least and mildly aqueously altered CM chondrites. Additionally, the $\beta$ alanine to glycine ratio correlates with the progressive degree of aqueous alteration, with Paris showing the lower $\beta$-alanine to glycine ratio for a $\mathrm{CM}$ chondrite. However, even if Paris represents an exceptional example of an almost unaltered CM, the 3.0 type has not yet been assigned. The scale proposed by Rubin et al. (2007) is then waiting for a hypothetical unaltered $\mathrm{CM}$ in order to attribute an absolute significance to this scale.

In order to better understand the origin of the observed amino acid abundances and distribution in chondritic meteorites, we should retrace the sequence of processes and events that led to it from the chemistry in interstellar and pre-cometary icy grains and then through the accretion of small bodies in the nascent solar system. In this perspective, an important tool is the study of interstellar ice evolution performed by laboratory experiments aimed to simulate the physical and chemical conditions in which these ices formed and evolved. Photo and thermal processing of icy mixtures originally composed of simple molecules (e.g., $\mathrm{H}_{2} \mathrm{O}, \mathrm{CH}_{3} \mathrm{OH}$, and $\mathrm{NH}_{3}$ in our experiments) leads to the formation of semi-refractory organic residues. These residues have often been considered as analogs or templates for the organic material synthetized in interstellar or pre-cometary icy grains that could be later incorporated into the small bodies of the solar system (e.g., Greenberg \& Yencha 1973; Agarwal et al. 1985; Allamandola et al. 1988; Briggs et al. 1992; Bernstein et al. 1995; Strazzulla 1997; Hudson \& Moore 2000; Muñoz Caro et al. 2004; Elsila et al. 2007; Nuevo et al. 2009; de Marcellus et al. 2017). The characteristics of these residues, such as the composition and the degree of molecular complexity, point to possible astrophysical implications also connected with prebiotic chemistry, in the case of an exogenous delivery scenario of organic matter on Earth (Oro 1961; Brack 2009; Martins et al. 2017).

A wide variety of organic compounds, some of them of biological relevance, is now reported to be present in these residues. The detected molecules include nucleobases (Nuevo et al. 2009, 2017), glycerol, urea, glycolic acid (Nuevo et al. 2010), hydantoin (de Marcellus et al. 2011a), glyceraldehyde (de Marcellus et al. 2015), various aldopentoses such as ribose, and a large set of sugar-related compounds (Meinert et al. 2016). Amino acids are a class of well-studied organic compounds in residues (Briggs et al. 1992; Kasamatsu et al. 1997; Bernstein et al. 2002; Muñoz Caro et al. 2002). Meinert et al. (2012) analyzed a residue produced by our group at the Institut d'Astrophysique Spatiale (IAS) in Orsay (France) using comprehensive two-dimensional gas chromatography coupled to time-offlight mass spectrometry (GC $\times$ GC-TOFMS), and identified 20 amino acids and 6 diamino acids. The same technique was used to measure the enantiomeric excesses of amino acids in residues produced in similar conditions by ultraviolet (UV) circularly polarized light irradiation at the SOLEIL synchrotron. It has been shown that this asymmetric UV irradiation indeed induces small (1\%-2\%) L-enantiomeric excesses (de Marcellus et al. 2011b; Modica et al. 2014). These experiments do have a crucial role in the investigation of the origin of the enantiomeric excesses of the L-form found in some meteoritic amino acids (see Myrgorodska et al. 2015 for a review).

In this work, we present three laboratory residues. We have analyzed two of them following the same experimental method (gas chromatography-mass spectrometry (GC-MS)) used for the $\mathrm{CM}$ chondrite Paris. A third one was previously analyzed by a different technique $(\mathrm{GC} \times \mathrm{GC}$-TOFMS $)$. We compare the abundances and molecular distribution of the amino acids in the three laboratory residues with previously published amino acid analyses of several CM chondrites (Ehrenfreund et al. 2001; Botta et al. 2002; Shimoyama \& Ogasawara 2002; Glavin et al. 2006, 2010; Martins et al. 2015). These meteorites have experienced various levels of aqueous processing and belong to petrological subtypes ranging from 2.0-2.8 (Rubin et al. 2007; Alexander et al. 2013). This comparison may provide insights into the origin of organic materials, in particular amino acids, that may have been retained inside primitive CM meteorites (Danger et al. 2016).

\section{Laboratory Experiments}

Three organic residues (named Residues 1, 2, and 3) and two separate blanks (Blanks 1 and 2) were prepared using the experimental setup at IAS. This setup is aimed to simulate the photo/thermo-chemical evolution of interstellar/pre-cometary ices, and it has already been fully described elsewhere (Nuevo et al. 2006, Nuevo et al. 2007; de Marcellus et al. 2011b). In brief, it consists of a high vacuum chamber operating at a pressure $<10^{-7}$ mbar, interfaced with a Fourier-transform infrared spectrometer that is used to monitor the sample characteristics and evolution. Inside the high vacuum chamber, a substrate window composed of magnesium fluoride $\left(\mathrm{MgF}_{2}\right)$, transparent in the IR range, is maintained at $77 \mathrm{~K}$. The vacuum chamber is connected to an introduction line kept at a pressure of a few $10^{-6}$ mbar, used for the gas mixture preparation. Different reservoirs connected to the introduction line hold the individual components of the mixture (water, methanol, and ammonia). These components are liquid at room temperature, however, only their vapors are used. These vapors are slowly 
admitted one by one into a mixing glass bottle, in the order of their increasing vapor pressure value, i.e., $\mathrm{H}_{2} \mathrm{O}, \mathrm{CH}_{3} \mathrm{OH}$, and $\mathrm{NH}_{3}$, and in the ratio of $\sim 2: 1: 1$, respectively. The relative proportions between the components are controlled by measuring their partial pressures at the introduction in the line. About 45 mbar of total gas mixture are prepared for each experiment, water being the limiting species because of its low vapor pressure compared to the other components. The gas mixture is stored for a few hours in the glass bottle for mixing. It is then finally admitted, through a micrometric valve, into the vacuum chamber, where it is deposited on the $\mathrm{MgF}_{2}$ substrate by freezing onto its cold surface. During a standard experiment, the gas mixture continuously flows and freezes onto the substrate for about $70 \mathrm{hr}$. The resulting solid ice sample qualitatively represents the composition of interstellar/precometary ices (Dartois 2005). Simultaneously with the deposition, the forming ice sample is irradiated by a UV photon source (a classical microwave stimulated hydrogen flow discharge lamp) to ensure its full and homogeneous irradiation, corresponding here to about one UV photon (Lya) per deposited molecule. This irradiation qualitatively simulates the UV irradiation field to which interstellar/pre-cometary ices are exposed for around $10^{5}-10^{6}$ years (Greenberg 1978; Hagen et al. 1979). During the irradiation, the molecules initially constituting the ice sample are photolyzed and new species and radicals are formed. At the end of the deposition/irradiation step, the ice sample is gradually warmed $\left(1 \mathrm{~K} \mathrm{minutes}^{-1}\right)$ to room temperature to allow the sublimation of volatiles, the diffusion of radical species, and their subsequent recombination (d'Hendecourt et al. 1982). Once at room temperature and still under high vacuum, a semi-refractory solid residue remains on the $\mathrm{MgF}_{2}$ window as recorded through infrared spectroscopy (see for example Muñoz Caro \& Schutte 2003 and de Marcellus et al. 2017). The window is then recovered in a clean environment in a horizontal laminar flow cabinet for further ex situ chemical analysis.

Residues 1 and 2 were prepared according to this standard procedure, while Residue 3 was obtained as a multilayered sample by repetitions of the standard procedure over 10 days in order to obtain a thicker residue and a larger amount of synthetized material. It has been isotopically ${ }^{13} \mathrm{C}$-labeled $\left(\mathrm{H}_{2} \mathrm{O}:{ }^{13} \mathrm{CH}_{3} \mathrm{OH}: \mathrm{NH}_{3}\right)$ but is qualitatively in compliance with Residues 1 and 2. Residue 3 was produced in a previous work of our group at IAS (de Marcellus 2010; Meinert et al. 2012).

In addition to the residue samples, two distinct blanks were also prepared, one obtained by deposition of the gas mixture $\left(\mathrm{H}_{2} \mathrm{O}: \mathrm{CH}_{3} \mathrm{OH}: \mathrm{NH}_{3}\right)$ without irradiation, followed by warm-up and recovery of the window at room temperature (Blank 1), and one by irradiation of the $\mathrm{MgF}_{2}$ window, without any gas deposition, followed by warm-up and recovery of the window also at room temperature (Blank 2). Both for the preparation of Residues 1 and 2 and for the blanks, we proceeded with the same time schedule ( $\sim 70 \mathrm{hr}$ of irradiation followed by $\sim 30 \mathrm{hr}$ of warm-up). The use of blanks allowed determining potential sources of contamination occurring at any step of the sample preparation (i.e., manipulation of the windows, gas mixture preparation, mixture deposition, mixture irradiation, and recovery procedure of the sample).

After the preparation protocol, all the samples were safely kept in argon-filled vessels to avoid oxidation and/or contamination. Residue 3 was analyzed at the Institute of Chemistry of Nice (France) and the protocol is fully described
Table 1

Average Amino Acid Abundances in Residue 1 and Residue 2

\begin{tabular}{lcc}
\hline \hline Amino Acid & $\begin{array}{c}\text { Abundance }^{\text {a }}(\mathrm{ng}) \text { in } \\
\text { Residue } 1\end{array}$ & $\begin{array}{c}\text { Abundance (ng) in } \\
\text { Residue } 2\end{array}$ \\
\hline Glycine & $1034 \pm 28$ & $249 \pm 19$ \\
D-alanine & $246 \pm 16$ & $109 \pm 8$ \\
L-alanine & $221 \pm 9$ & $107 \pm 9$ \\
$\beta$-alanine & $136 \pm 5$ & $37 \pm 3$ \\
D,L- $\alpha$-ABA & 71 & $<36$ \\
\hline
\end{tabular}

Notes.

a The associated errors are based on the standard deviation of the average value between eight separate measurements $(N)$ with a standard error $\delta x=\sigma x . N^{-1 / 2}$.

${ }^{\mathrm{b}}$ Optically pure standard not available for enantiomeric identification.

in Meinert et al. (2012). Residues 1 and 2, and Blanks 1 and 2 were analyzed at Imperial College London (UK), following the same experimental method for the analysis of amino acids used by our group for the CM chondrite Paris. In the following, we describe the protocol for the analysis of Residues 1 and 2, and Blanks 1 and 2.

Each sample was washed with $50 \mu \mathrm{L}$ of high-performance liquid chromatography (HPLC)-grade water, which was transferred to small test tubes ( $3 \mathrm{ml}$ each), and dried under vacuum. They were then carried through the same protocol of $6 \mathrm{M} \mathrm{HCl}$ acid vapor hydrolysis $\left(150^{\circ} \mathrm{C}\right.$ for $\left.3 \mathrm{hr}\right)$, desalting on a cation exchange resin (with amino acids elution using $5 \mathrm{ml}$ of $2 \mathrm{M}$ ammonium hydroxide), trifluorocetic anhydride/isopropanol derivatization and GC-MS analyses used to characterize the amino acids present in the Paris meteorite (Martins et al. 2015). The GC-MS analyses were performed on a Perkin Elmer Clarus SQ8S MS and a Clarus $580 \mathrm{GC}$, with a helium flow of $1 \mathrm{ml}$ minutes $^{-1}$. The MS source was held at $230^{\circ} \mathrm{C}$, while the MS transfer line and injection port were held to $220^{\circ} \mathrm{C}$. Separation of the D, L-amino acids was achieved using a Chirasil-L-Val column $(50 \mathrm{~m} \times 0.25 \mathrm{~mm} \mathrm{ID} \times 16 \mu \mathrm{m}$ film thickness $)$ from Alltech. The oven temperature was set for 5 minutes at $65^{\circ} \mathrm{C}$, increased by $2^{\circ} \mathrm{C}$ minutes ${ }^{-1}$ to $80^{\circ} \mathrm{C}$ and held for 5 minutes, increased to $100^{\circ} \mathrm{C}$ by $1^{\circ} \mathrm{C}$ minutes ${ }^{-1}$, increased to $200^{\circ} \mathrm{C}$ by $2^{\circ} \mathrm{C}$ minutes ${ }^{-1}$ and held for 10 minutes, and increased by $10^{\circ} \mathrm{C}$ minutes $^{-1}$ to $220^{\circ} \mathrm{C}$ and held for 5 minutes. The amino acids were identified by comparison of the retention times and mass fragmentation pattern with known amino acid standard mixtures. An internal standard was used (D, L-2-aminohexanoic acid). Amino acids were quantified by peak area integration of the corresponding ion fragment. The areas were then converted to abundances (presented in Table 1) using calibration curves, which were created by plotting the ratio of the amino acid standard/internal standard target ion peak area versus the mass of amino acid standard injected into the column. All the glassware used at Imperial College London was sterilized by wrapping in aluminum foil and baked at $500^{\circ} \mathrm{C}$ for at least $6 \mathrm{hr}$. This protocol is fully described in Martins et al. (2015), including the molecular ions used for quantification and identification of the amino acids.

\section{Results and Discussion}

\subsection{Amino Acid Abundance and Distribution}

Residues 1 and 2, and Blanks 1 and 2 were analyzed by GCMS. The single ion GC-MS chromatograms obtained for the two residues are shown in Figures 1 and 2, respectively. The 


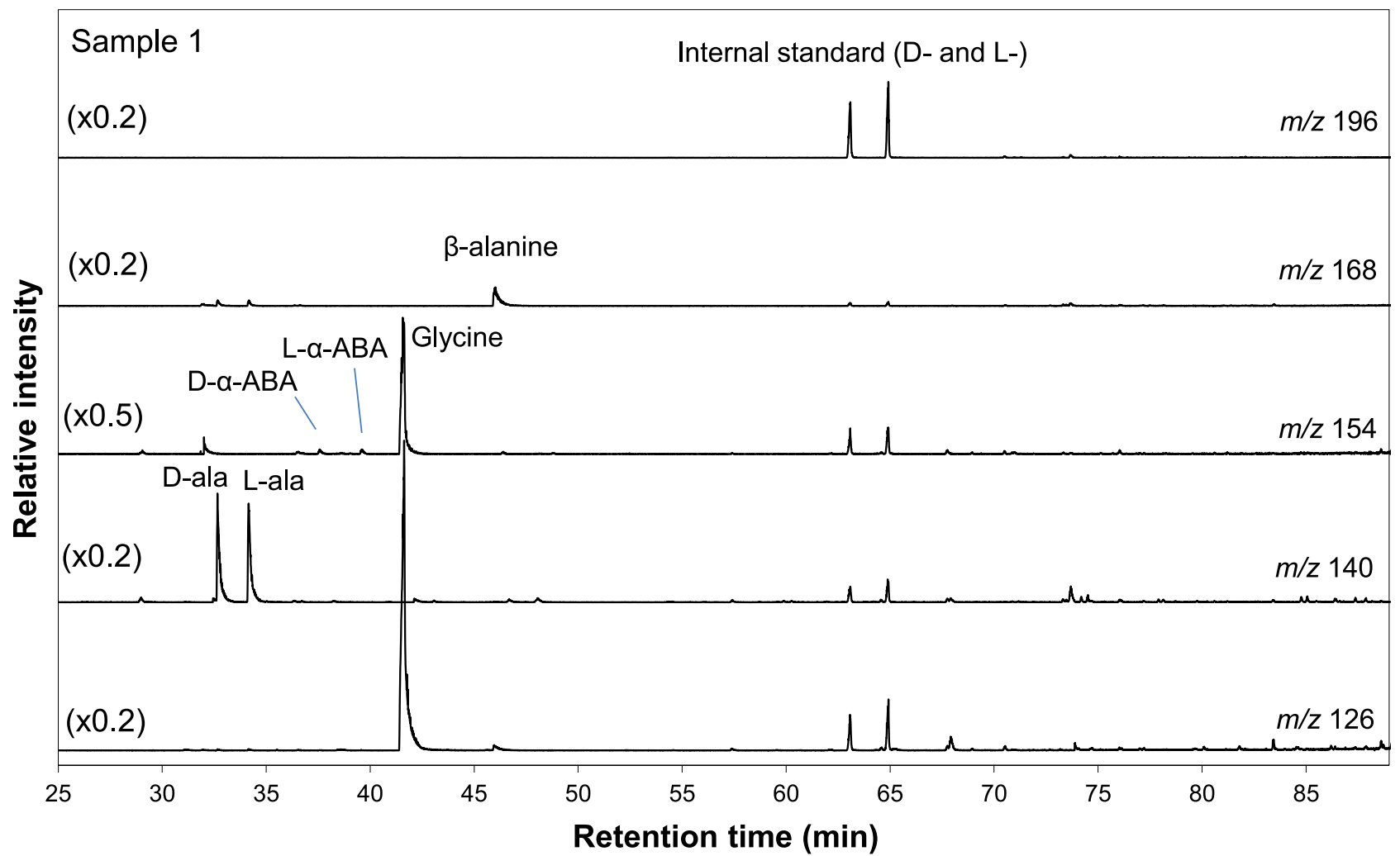

Figure 1. Single ion GC-MS chromatograms $(\mathrm{m} / \mathrm{z} 126,140,154,168,196)$, in the $25-90$ minutes region showing the detection of the derivatized $(N-$ TFA, $O$ isopropyl) glycine, D-alanine (D-ala), L-alanine (L-ala), D- $\alpha$-aminobutyric acid (D- $\alpha$-ABA), L- $\alpha$-aminobutyric acid (L- $\alpha$-ABA), and $\beta$-alanine in Residue 1 . The peaks corresponding to the derivatized internal standard are visible in the single ion traces. Relative intensity (zoom $\times 0.2$, and zoom $\times 0.5$ for $\mathrm{m} / \mathrm{z} 154$ ) is adapted for clarity reasons.

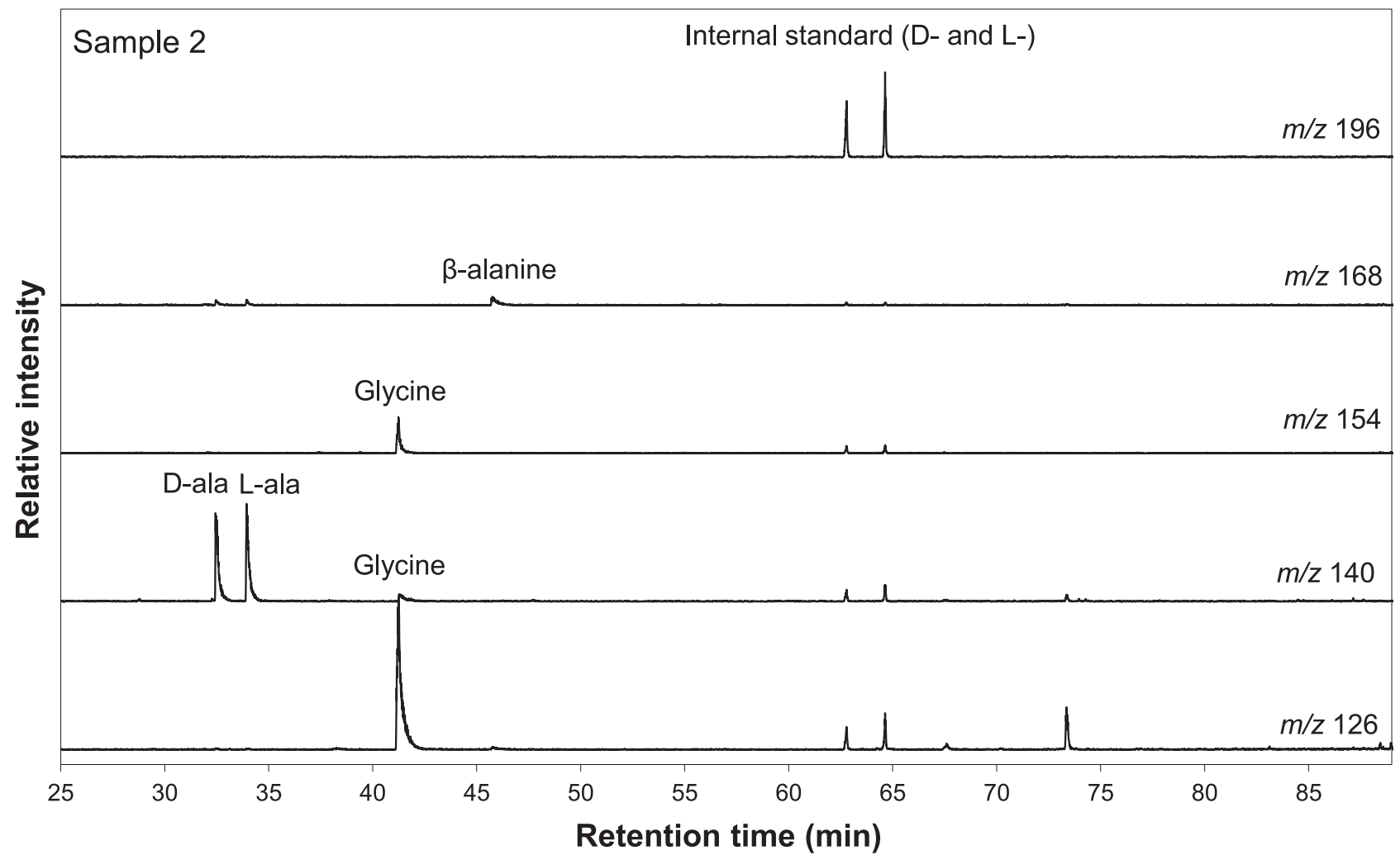

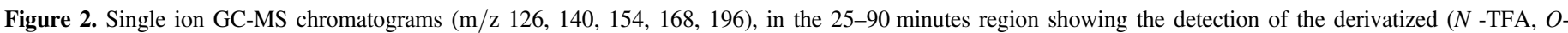

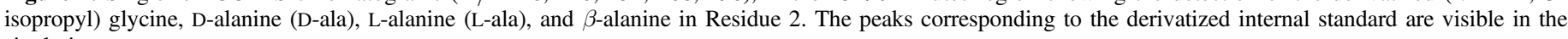
single ion traces. 
peaks indicate the elution of the ions of interest at selected $\mathrm{m} / \mathrm{z}$ values (126, 140, 154, 168, and 196).

Amino acids detected in Residue 1 include glycine (gly), D-alanine (D-ala), L-alanine (L-ala), D- $\alpha$-aminobutyric acid (D- $\alpha$-ABA), L- $\alpha$-aminobutyric acid (L- $\alpha$-ABA), and $\beta$-alanine ( $\beta$-ala). In the case of $\alpha$-ABA, L- and D-optically pure standards were not available for the enantiomeric identification and the order of elution of $\mathrm{D}-\alpha$-ABA and $\mathrm{L}-\alpha$-ABA was tentatively assigned considering the order of elution of the enantiomers (D first and L after) when using a Chirasil- L-Val column. Amino acids detected in Residue 2 include glycine, D-alanine, L-alanine, $\beta$-alanine, and an upper limit of D,L- $\alpha$ ABA. Additional amino acids such as glutamic acid, $\gamma$-ABA, aspartic acid, etc. were searched for in both residues but they were not detected, either because they were not formed and/or they were present below the detection limit of the GC-MS (10 pg of amino acids).

The two blanks do not show any amino acids (Figure 3, top and bottom). This testifies to the absence of sources of external contamination of amino acids above the detection limit of the GC-MS. Furthermore, Blank 1 (no UV irradiation) contains no amino acids, which shows that irradiation is responsible for the formation of amino acids and not thermal processing by itself.

The abundance of glycine, D- and L-alanine, $\beta$-alanine, and $\mathrm{D}, \mathrm{L}-\alpha$-aminobutyric acid were determined. Table 1 lists the average amino acid abundances (in nanograms) of eight separate measurements together with the associated standard errors for Residues 1 and 2. In the case of Residue 3, for comparison, we report in Table 2 the abundances relative to glycine (gly $=100$, arbitrary unit) of the same amino acids plus a set of isomers that are of interest for this work (data from Meinert et al. 2012).

We observe that in the three residues, glycine is the most abundant amino acid, followed by D- and L-alanine and then by $\beta$-alanine. D,L- $\alpha$-ABA is the least abundant among the detected amino acids. For this latter, the low abundance prevents the estimate of the associated standard error for Residue 1, while only upper limits $(<36 \mathrm{ng})$ can be given for Residue 2 (Table 1). For Residue 3, D- and L- $\alpha$-ABA were separated (Table 2). Results between Residues 1 and 2 agree well in terms of amino acid distribution, even if their absolute abundances are systematically higher for Residue 1. Residue 1 resulted in fact in a larger amount of material than Residue 2. This difference can be explained by the variability of some parameters during the sample preparation that caused a different amount of organic material synthetized. Such parameters include the deposition rate of the mixture and the flux of the UV irradiation lamp, which are difficult to maintain strictly constant over the time needed for the production of both residues.

If we compare the amino acid content of Residues 1 and 2 with the one of the Paris meteorite presented in Martins et al. (2015), which were analyzed with the same experimental method by our group, we note that the number of different amino acids detected in the residues is smaller than in Paris, and with a restricted distribution limited to $\alpha$ - and $\beta$-amino acids only. This is mostly due to the difference between the total quantity of organic matter available in these residues as compared to the soluble organic matter (SOM) extracted from the Paris meteorite. In a standard residue, we estimate this quantity between 50 and $100 \mu \mathrm{g}$ (de Marcellus 2010). In the case of Paris, we estimate it to be at least $1.2 \mathrm{mg}$ for the amount of matrix material at our disposal $(\sim 200 \mathrm{mg})$.

\subsection{Beta-alanine to Glycine Ratio}

We considered the $\beta$-alanine to glycine ratio for our three residues and compared these values to that of a set of CM chondrites presenting an increasing degree of aqueous alteration (Figure 4). The meteorites we have selected include all the $\mathrm{CM}$ chondrites for which the $\beta$-alanine to glycine ratio as well as the hydrothermal scale (petrological subtypes) are known (Rubin et al. 2007; Alexander et al. 2013). Indeed, a study of the correlation between the amino acid distribution and aqueous alteration can be accurate if both these parameters are known. These meteorites are listed in Table 3. They have experienced levels of aqueous alteration ranging from 2.7 (for the least altered) to 2.0 (for the more altered). The petrologic subtype of the Paris meteorite is based on the hydrothermal scale designated by Rubin et al. (2007). The petrologic subtypes of all other CM chondrites is determined by Alexander et al. (2013), and are based on correlations between bulk $\mathrm{H}$ and $\mathrm{N}$ isotopic compositions and the petrologic criteria of Browning et al. (1996) and Rubin et al. (2007).

In Figure 4, the $\beta$-alanine to glycine ratio for Residues 1,2 , and 3 are placed at the extreme left of the $x$-scale, which would indicate no aqueous alteration. This figure shows an increasing trend of the $\beta$-alanine to glycine ratios from the residues toward the different $\mathrm{CM}$ chondrites. The residue values fit the trend followed by the CM chondrites, whose values increase with the increasing degree of aqueous alteration.

\subsection{Alpha, Beta, and Gamma Amino Acid Distribution}

For the study of the distribution of the different groups of amino acids, we considered the list of amino acids detected in Residue 3 , analyzed by GC $\times$ GC-TOFMS. The analysis by classical GC-MS of Residues 1 and 2 did not provide a measurable concentration of $\gamma$-amino acids for their analysis in relation to $\alpha$ - and $\beta$-amino acids, because of the rather limited amount of organic material of the residues available for the analysis.

In Residue 3, 20 different amino acids were previously detected and relative abundances with respect to glycine were obtained. Among the amino acids detected in Residue 3, we considered a consistent set of amino acids ( $\alpha$-ABA, $\beta$-ABA, $\beta$ $\mathrm{AIB}$, and $\gamma$-ABA) containing 4-carbon atoms and spanning the $\alpha$-, $\beta$-, and $\gamma$-groups. In Table 2 , we report the relative abundances of the considered 4-carbon amino acids with respect to glycine for Residue 3, as measured in Meinert et al. (2012).

We considered the relative abundances of 4-carbon $\alpha-, \beta$-, and $\gamma$-amino acids with respect to the total amino acid abundances ( $\alpha$ - plus $\beta$ - plus $\gamma$-amino acids) obtained for Residue 3, and compared these values to those of the CM chondrites with increasing degrees of aqueous alteration for which data are available (Figure 5). This group includes the same list of CM chondrites already used for Figure 4. Also in this case, the least aqueously altered CM chondrites are on the left of the plot, while the most altered ones are on the right. Residue 3 is placed at the "zero" of the aqueous alteration scale, just before the Paris meteorite, reported to be the least aqueously altered CM. 

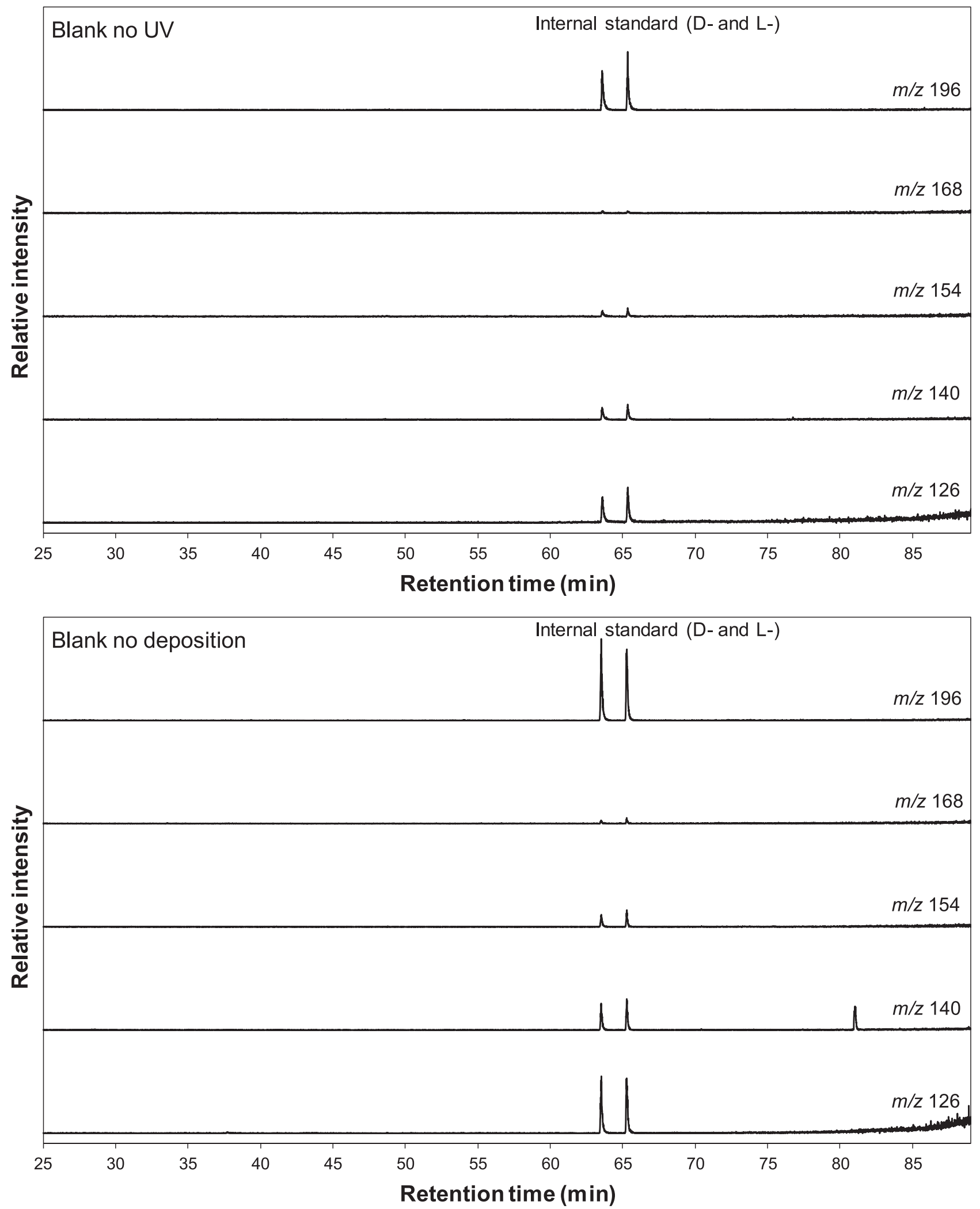

Figure 3. Single ion GC-MS chromatograms (m/z 126, 140, 154, 168, 196), in the 25-90 minutes region of the blanks obtained from deposition of the gas mixture without UV irradiation (top) and from UV irradiation without any deposition (bottom), respectively. The peaks corresponding to the derivatized internal standard are visible in the single ion traces.

As already noted in Martins et al. (2015), the comparison between the relative distributions for each of the individual 4-carbon $\alpha-, \beta$-, and $\gamma$-amino acid sets, shows that the least and moderate aqueously altered CM chondrites (i.e., Paris, LON 94102, Murchison, Murray, Y-791198, and LEW 90500) have much higher relative abundances of 4-carbon $\alpha$-amino acids 
Table 2

Relative Abundances to Glycine $($ Gly $=100)$ for Amino Acids of Interest Detected in Residue 3 (from Meinert et al. 2012)

\begin{tabular}{lc}
\hline \hline Amino Acid & Abundance in Residue 3 \\
\hline Glycine & 100 \\
D-alanine & 31.3 \\
L-alanine & 32.1 \\
$\beta$-alanine & 2.90 \\
D- $\alpha$-ABA & 0.36 \\
L- $\alpha$-ABA & 0.37 \\
$\beta$-ABA & 0.02 \\
$\gamma$-ABA & 0.17 \\
$\beta$-AIB & 0.22 \\
\hline
\end{tabular}

than $\beta$ - and $\gamma$-amino acids with respect to the most aqueously altered ones (i.e., Mighei, Nogoya, ALH 83100, MET 01070, and SCO 06043). The relative abundances of $\alpha-, \beta$-, and $\gamma-$ amino acids for Residue 3 match the behavior of the least aqueously altered $\mathrm{CM}$ chondrites. In particular, the relative abundance of 4-carbon $\alpha$-amino acids (0.64) is high and comparable to the average value of these least aqueously altered $\mathrm{CM}$ chondrites. The relative abundance of 4 -carbon $\beta$ amino acids $(0.21)$ is in the average between the $\beta$-values of all the considered $\mathrm{CM}$ chondrites. Finally, the relative abundance of 4-carbon $\gamma$-amino acids (0.15) is low and comparable to that of the least aqueously altered CM chondrites. We observe that the distribution of $\alpha$ - and $\gamma$-amino acids seems to vary with the degree of aqueous alteration, although without following a clear trend. In particular, $\alpha$-amino acids dominate the low aqueous alteration range, $\gamma$-amino acids appear to be higher in the high aqueous alteration ones, while $\beta$-amino acids are rather equally distributed in the whole range and do not seem to be affected by the increase of aqueous alteration.

\section{Astrophysical Implications}

The study of the content and relative distribution of amino acids in the laboratory residues described in this work provides key information about the general formation mechanism of these molecules in astrophysical environments and their evolution in the small bodies of the solar system.

Indeed, many organic molecules, including amino acids, can be formed within the materials synthetized via photo- and thermo-chemistry of ices (d'Hendecourt et al. 1986; Bernstein et al. 2002; Muñoz Caro et al. 2002; Elsila et al. 2007, 2012). Several molecules do readily form in the ice at low temperatures as shown for glycine (Bossa et al. 2009), diamino acids, dipeptides, urea, and aldehydes (Kaiser et al. 2014; Kaiser 2015) by in situ infrared spectroscopy. Moreover, thermal processing enhances the production of these molecules via radical diffusion and recombination, allowing their detection at room temperature using GC-MS techniques. In particular, numerous amino acids have been reported in laboratory residues, generally after acid hydrolysis (Bernstein et al. 2002; Muñoz Caro et al. 2002; Nuevo et al. 2008; Meinert et al. 2012). Acid hydrolysis is a protocol that allows the identification of bound amino acids present in larger macromolecular structures by breaking their chemical bonds. However, amino acids are detected in laboratory residues both as free ones (i.e., in non-hydrolyzed samples) and as bound ones (i.e., in hydrolyzed samples), and they are qualitatively similar although more abundant in the hydrolyzed samples
(Nuevo et al. 2008). The acid hydrolysis may also lead to the additional formation of amino acids from initial amino acid precursors previously synthesized by irradiation. Aminonitriles have been proposed as amino acid precursors in astrophysical conditions (Elsila et al. 2007), as they have been detected in the interstellar gas phase (Belloche et al. 2008). Aminoacetonitrile, which has been synthetized in astrophysical-like conditions by irradiation of ice analogs containing acetonitrile and ammonia (Danger et al. 2011), may be further hydrolyzed to glycine.

It has been shown that the formation of amino acids may occur via multiple pathways, some major and many minor others existing as well (Elsila et al. 2007; Hudson et al. 2008). This observation suggests that their formation is not rigidly dependent on a well-defined ice composition and specific chemical pathways, but may occur under a variety of conditions, as it must be the case in a natural environment. Different experimental parameters that could influence the variety, quantity, and distribution of amino acids in laboratory residues have been explored, such as the irradiation time, the temperature, the ice mixture composition, the photon dose per molecule as well as the irradiation agent ( $\mathrm{MeV}$ protons), and the substrate for the ice deposition (Nuevo et al. 2008; Muñoz Caro et al. 2014). It was found that the total quantities and distribution of amino acids depend only slightly on the experimental parameters explored. The important requirement to form such molecules is that the starting ice mixtures must contain the four elements $\mathrm{C}, \mathrm{H}, \mathrm{O}$, and $\mathrm{N}$ and roughly follow relative cosmic abundances. In addition, recent experiments in which the relative amount of water in the initial ice mixture is varied by a factor of 3 , clearly show that varying the ice composition does not influence much the chemistry of the obtained organic residues (Fresneau et al. 2017). Increasing significantly the radiation dose leads to insoluble organic residues (de Marcellus et al. 2017). However, using the protocol described in the present work only soluble organic residues are produced.

In space, amino acids could have been formed by a general process of photo- and thermo-chemistry of ices, then be retained during the accretion into planetesimals, and later accreted into asteroids (Ehrenfreund et al. 2001). Due to the unevenly irradiation of the protoplanetary disc, we assume that processed ices had a nonhomogenous composition, depending on their residing region. However, after dynamical processes in the disk, these ices have likely been mixed (Bergin et al. 2007; Caselli \& Ceccarelli 2012; Ciesla \& Sandford 2012). As a direct consequence, the composition of the asteroids is also nonhomogeneous. This may explain why a single CM parent body contains regions with different amounts of water and different contents and distributions of amino acids and other organics.

Complementary, amino acids could also be formed inside the parent bodies after aqueous alteration, as shown by the increased levels of amino acids in more aqueously altered carbonaceous meteorites (Sephton 2002; Martins et al. 2007; Glavin et al. 2010; Aponte et al. 2017). However, these mechanisms may require the presence of precursors that can be formed after processing of interstellar ices. We note that also in this case, aqueous alteration was not a homogeneous process because it depends on the local amount of embedded radioactive nuclei and on the size of a given parent body, which strongly affects its cooling rate. 


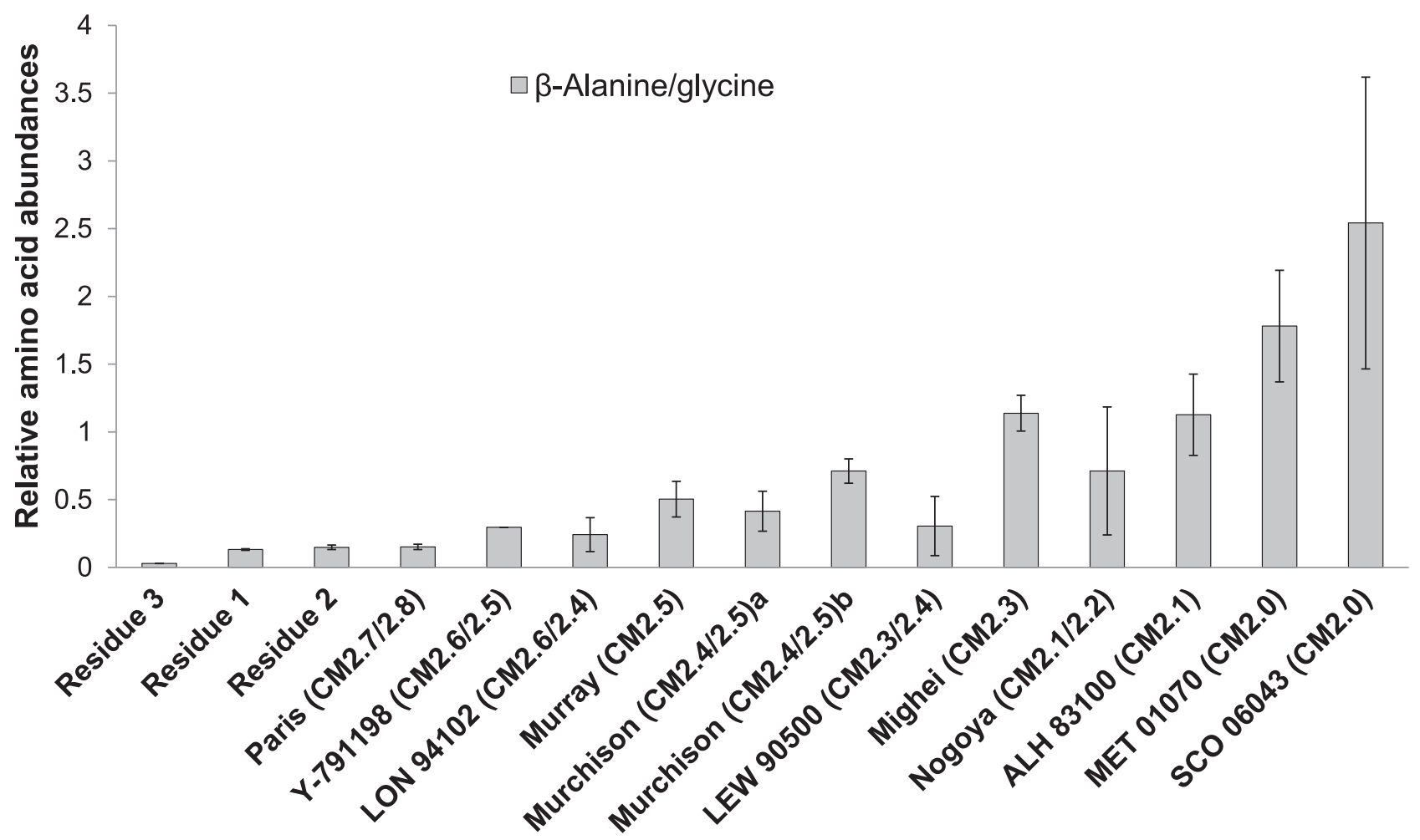

Increasing aqueous alteration

Figure 4. Relative abundance of $\beta$-alanine/glycine for Residues 1, 2, and 3, and CM chondrites with increasing degrees of aqueous alteration. The petrologic subtype of the Paris meteorite is based on the hydrothermal scale designated by Rubin et al. (2007). The petrologic subtypes of all other CM chondrites were determined by Alexander et al. (2013), and are based on correlations between bulk H and N isotopic compositions and the petrologic criteria of Browning et al. (1996) and Rubin et al. (2007) (Table 3). The $\beta$-alanine/glycine position for Residues 1, 2 and 3 indicates no aqueous alteration. The relative abundance of $\beta$-alanine/glycine includes data for Residues 1 and 2 (Table 1, this study), Residue 3 (Table 2, this study; from Meinert et al. 2012), and for the CM chondrites listed in Table 3 . The uncertainties are obtained by standard propagation calculation.

Table 3

List of the CM Meteorites Considered in this Study along with Their Petrologic Subtypes and References Used for the $\beta$-Alanine to Glycine Ratio

\begin{tabular}{lcl}
\hline \hline Meteorite & $\begin{array}{c}\text { Petrologic } \\
\text { Subtype }^{\mathrm{a}}\end{array}$ & References \\
\hline Paris & CM2.7/2.8 & Martins et al. (2015) \\
Y-791198 & CM2.6/2.5 & Shimoyama \& Ogasawara (2002) \\
LON 94102 & CM2.6/2.4 & Glavin et al. (2010) \\
Murray & CM2.5 & $\begin{array}{c}\text { Ehrenfreund et al. (2001), Botta } \\
\text { et al. (2002) }\end{array}$ \\
Murchison (a) & CM2.4/2.5 & Glavin et al. (2010) \\
Murchison (b) & CM2.4/2.5 & Glavin et al. (2006) \\
LEW 90500 & CM2.3/2.4 & Glavin et al. (2006) \\
Mighei & CM2.3 & Botta et al. (2002) \\
Nogoya & CM2.1/2.2 & Botta et al. (2002) \\
ALH 83100 & CM2.1 & Glavin et al. (2006) \\
MET 01070 & CM2.0 & Glavin et al. (2010) \\
SCO 06043 & CM2.0 & Glavin et al. (2010) \\
\hline
\end{tabular}

Note.

${ }^{a}$ Petrologic subtypes are determined by Alexander et al. (2013), except for Paris (Rubin et al. 2007).

Moreover, aqueous alteration can be both synthetic and destructive, and there is likely some competition between these two tendencies. Extensive aqueous alteration on the meteorite parent body may result in the decomposition of $\alpha$-amino acids (Botta et al. 2007; Martins et al. 2007). It may also result in hydrolysis of lactams, leading to the synthesis of $\beta$ - and $\gamma$ amino acids (Cooper \& Cronin 1995) and/or it may favor the Michael addition reaction to form $\beta$-amino acids (Cronin \& Chang 1993).

We consider our residues as analogs of the organic material that forms by photo- and thermo-chemical processing of icy grains before accretion into planetesimals and before any further aqueous alteration on the asteroidal parent bodies. This is the situation in the vacuum of space but also in the laboratory, because the residues do form by sublimation of the irradiated ice samples in a dynamic high vacuum chamber, in which liquid water is never present. Indeed, the similarity between these laboratory residues and the SOM found in carbonaceous chondrites was recently proposed (Danger et al. $2013,2016)$ using ultra high-resolution mass spectrometry for the analysis of laboratory residues having the same composition and preparation procedure than the ones presented here.

We compared the amino acid content and distribution of three residues with the one of different $\mathrm{CM}$ chondrites. From this comparison, some similarities are evident. First, the amino acid distribution (Table 1) is analogous to that of Paris and other CM chondrites (Martins et al. 2015), with the simplest amino acids as the most abundant, and abundances tending to decrease as the number of carbon (C) atoms increases (glycine (2C) $>$ D, L -alanine $(3 \mathrm{C})>\mathrm{D}, \quad$ L $\alpha$-ABA (4C)). The decreasing trend of amino acid abundances with the number of carbon atoms is a characteristic already observed in residues 


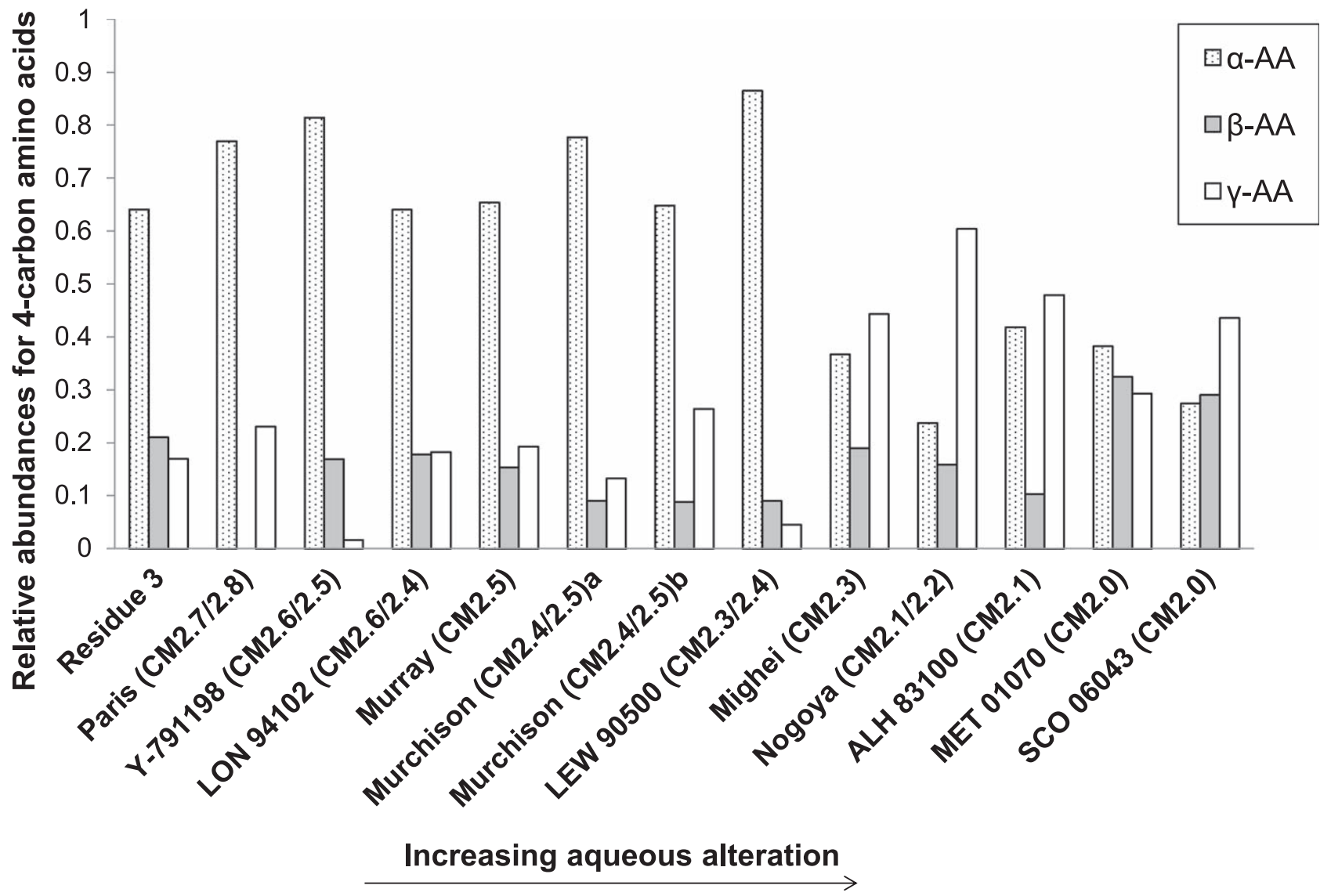

Figure 5. Relative abundances (total $=\alpha$-plus $\beta$-plus $\gamma$-amino acids) for the 4-carbon amino acid content of $\alpha$-(dots), $\beta$-(gray), and $\gamma$-(white) amino acids for Residue 3 and $\mathrm{CM}$ chondrites with increasing degrees of aqueous alteration. The petrologic subtype of the Paris meteorite is based on the hydrothermal scale designated by Rubin et al. (2007). The petrologic subtypes of all other CM chondrites were determined by Alexander et al. (2013), and are based on correlations between bulk $\mathrm{H}$ and $\mathrm{N}$ isotopic compositions and the petrologic criteria of Browning et al. (1996) and Rubin et al. (2007). The relative abundances include data for Residue 3 (Table 2, this study; from Meinert et al. 2012) and for the CM chondrites listed in Table 3.

produced by our group (Nuevo et al. 2008; Meinert et al. 2012; Modica et al. 2014). It is a signature of the abiotic synthesis of these molecules that contrasts with the observed frequency of naturally occurring amino acids in living organisms.

Hydrothermal processes, in particular aqueous alteration, are thought to have impacted both the soluble and the insoluble organic matter in the parent asteroids (Martins et al. 2015; Vinogradoff et al. 2017, 2018; Yabuta et al. 2007). According to the extent of the asteroidal aqueous alteration experienced, the CM chondrites present different chemical characteristics, also reflected in the amino acid distribution. A parameter that seems to correlate with the degree of aqueous alteration is the $\beta$-alanine to glycine ratio, which has been proposed as an indicator for the extent of parent body aqueous alteration (Glavin et al. 2006). In our comparison with CM chondrites, we placed the $\beta$-alanine to glycine ratio of our residues at the extreme left of the aqueous alteration scale (Figure 4), in a position that indicates that no aqueous alteration has been taking place within the laboratory residue. Indeed, after their production, our residues are kept in an argon-filled stainlesssteel vessel until their extraction and thus not exposed to liquid water interaction. From Figure 4 we observe that the $\beta$-alanine to glycine ratio naturally assumes its lowest value in the case of the residues, then it increases toward the different $\mathrm{CM}$ chondrites with increasing aqueous alteration. Here, the residues represent an ideally unaltered organic material. Their presence allows extending downward the scale of aqueous alteration for CM meteorites and fixes a zeropoint that would otherwise be missing. This zeropoint would correspond to the pre-accretional unaltered material of the presolar cloud.

The observed trend for the $\beta$-alanine to glycine ratio with the increasing degree of aqueous alteration can be considered as an indication that may help to establish a link between the generic formation pathways of complex organic molecules, in particular amino acids, and soluble organic materials in primitive meteorites. This link may be found in the photoand thermo-processing of interstellar/pre-cometary icy grains in the absence of any possible aqueous alteration due to the physical conditions encountered at the place of their formation.

We follow Botta et al. (2007), Glavin et al. (2006, 2010) and Martins et al. $(2007,2015)$ in assuming that aqueous alteration in chondritic meteorites affects the distribution of amino acids of different classes $(\alpha, \beta$, and $\gamma)$. From the comparison between $\alpha$-, $\beta$-, and $\gamma$ - 4-carbon amino acids in Figure 5, it can be seen that the relative abundance of $\alpha$-amino acids decreases while that of $\gamma$-amino acids increases as the meteorites have faced more aqueous alteration. In fact, $\beta$ - and $\gamma$-amino acids could result from the degradation of $\alpha$-amino acids. All these mechanisms can coexist, although their individual role is not yet fully understood.

We found another important clue to support the idea that aqueous alteration affects the distribution of amino acids in more altered meteorites. Our results show a good similarity between the relative abundances of amino acids of the $\alpha-, \beta-$, 
and $\gamma$-groups present in the laboratory residues and in the least aqueously altered CM chondrites (Figure 5). These similarities offer some indications about the formation and evolution of the meteoritic amino acids, as already noted by Elsila et al. (2016). Our interpretation is that a relationship is possible between the amino acids present in very primitive meteorites (such as Paris, a CM2.7/2.8) and those produced on icy grains prior to the formation of planetesimals. In this scheme, fully primitive organic materials may remain preserved in the least altered part of the CM meteorites, from which Paris may be an exceptional example (Piani et al. 2017). We thus suggest that some meteoritic amino acids are a product of the ice evolution that occurred in the late stage of the parent molecular cloud or in the protoplanetary disk, by photo-irradiation and thermal processing prior to their incorporation in the asteroids as well as in comets, a scheme that is described by Ciesla \& Sandford (2012). This hypothesis is further supported by the results from Nakamura-Messenger et al. (2006) with the detection, in the primitive carbonaceous Tagish-Lake meteorite, of nanoglobules rich in organic carbon and presumed to be the remnants of photochemical processes on interstellar ices. Similar structures have been also evidenced in some fragments of the Paris meteorite matrix, for which vibrational infrared microspectroscopy shows large amounts of organic material concentrated in tiny fragments embedded in the matrix material (Merouane et al. 2012). In addition, the very primitive nature of these fragments is supported by the comparison between their infrared spectra with the ones of some solid-state features observed in two molecular clouds' lines of sight $\left(\mathrm{SgrA}^{*}\right.$ and GSC3), tracing, by definition, non-aqueously altered materials as discussed by Merouane et al. (2012).

\section{Conclusions}

In order to compare laboratory and meteoritic organic materials, we have considered three residues produced by our group. Two of them were analyzed following the same experimental method for the analysis of amino acids used by our group for the CM chondrite Paris. We detected glycine, Dand L-alanine, $\beta$-alanine, and $\mathrm{D}, \mathrm{L}-\alpha$-ABA, presenting decreasing abundances with increasing number of carbon atoms. The $\beta$-alanine to glycine ratio is similar to the one of Paris, the lowest ever measured ratio in CM chondrites, and correlates well with the increasing degree of aqueous alteration of different CM chondrites. The abundances of the amino acids from a third equivalent residue, previously produced by our group and analyzed using GC $\times$ GC-TOFMS, were also considered. The relative distribution of 4-carbon $\alpha-, \beta$-, and $\gamma$-amino acids is in agreement with that of the least aqueously altered CM chondrites.

The similarities observed between amino acids in the three laboratory residues and in primitive meteoritic materials provide insights about the formation and the evolution of the meteoritic amino acids. These results may expand our knowledge about the different mechanisms that could have contributed to the synthesis of these molecules and support the hypothesis of the formation process for complex organic molecules, in particular amino acids, from photo- and thermoprocessing of icy grains, prior to accretion in primitive solar system minor bodies. This formation process is complementary to other mechanisms but more comprehensive, as it can explain the presence of amino acids of different structural groups $(\alpha-$, $\beta-, \gamma$, etc.) at once and does not require specific starting conditions. According to this scenario, cosmic ices processing can be considered as an important source for the initial organic matter in our solar system prior to further planetary processes.

Z.M. acknowledges funding by the Royal Society (grant UF080820 and grant UF130324). Louis d'Hendecourt acknowledges the continual support of the French Space Agency (CNES) on the ice irradiation experiments as well as the support of the French national program PCMI. The authors acknowledge Pierre de Marcellus for preparing Residue 3. This work has been supported by the French government, through the UCAJEDI Investments in the Future project managed by the National Research Agency (ANR), reference number ANR-15-IDEX-01 as well as by the ANR RAHIIA-SOM, reference number ANR16-CE-2015-01. Finally, we wish to specially thank the referee for the detailed remarks and suggestions, which significantly improved the quality of this manuscript.

\section{ORCID iDs}

P. Modica (iD https://orcid.org/0000-0003-3002-8285

Z. Martins (iD https://orcid.org/0000-0002-5420-1081

\section{References}

Agarwal, V. K., Schutte, W., Greenberg, J. M., et al. 1985, OrLi, 16, 21 Alexander, C. M. O. 'D., Howard, K. T., Bowden, R., et al. 2013, GeCoA, 123,244

Allamandola, L. J., Sandford, S. A., \& Valero, G. J. 1988, Icar, 76, 225

Aponte, J., Elsila, J. E., Glavin, D. P., et al. 2017, ESC, 1, 3

Belloche, A., Menten, K. M., Comito, C., et al. 2008, A\&A, 482, 179

Bergin, E. A., Aikawa, Y., Blake, G. A., et al. 2007, in Protostars and Planets V, ed. B. Reipurth, D. Jewitt, \& K. Keil (Tucson, AZ: Univ. Arizona Press), 751

Bernstein, M. P., Dworkin, J. P., Sandford, S. A., et al. 2002, Natur, 416, 401 Bernstein, M. P., Sandford, S. A., Allamandola, L. J., et al. 1995, ApJ, 454,327

Bertaux, J.-L., \& Lallement, R. 2017, MNRAS, 469, 646

Blanchard, I., Gounelle, M., Bourot-Denise, M., \& Kearsley, A. 2011, M\&PSA, 74,5322

Bossa, J.-B., Duvernay, F., Theulé, P., et al. 2009, A\&A, 506, 601

Botta, O., Glavin, D. P., Kminek, G., et al. 2002, OLEB, 32, 143

Botta, O., Martins, Z., \& Ehrenfreund, P. 2007, M\&PS, 42, 81

Brack, A. 2009, NatGe, 2, 8

Brearley, A. J. 2006, in Meteorites and the Early Solar System II, ed.

D. S. Lauretta \& H. Y. McSween, Jr. (Tucson, AZ: Univ. Arizona Press), 584

Briggs, R., Ertem, G., Ferris, J. P., et al. 1992, OLEB, 22, 287

Browning, L. B., McSween, H. Y., \& Zolensky, M. E. 1996, GeCoA, 60, 2621 Burton, A. S., Stern, J. C., Elsila, J. E., et al. 2012, Chem. Soc. Rev., 41, 5459 Caselli, P., \& Ceccarelli, C. 2012, A\&ARv, 20, 56

Choe, W. H., Huber, H., Rubin, A. E., et al. 2010, M\&PS, 45, 531

Ciesla, F. J., \& Sandford, S. A. 2012, Sci, 336, 452

Cooper, G. W., \& Cronin, J. R. 1995, GeCoA, 59, 1003

Cronin, J. R., \& Chang, S. 1993, in The Chemistry of Life's Origins, ed. J. M. Greenberg, C. X. Mendoza-Gomez, \& V. Pirronello (Dordrecht: Kluwer), 209

Cronin, J. R., Cooper, G. W., \& Pizzarello, S. 1995, AdSpR, 15, 91

Cronin, J. R., Pizzarello, S., Cruikshank, D. P., et al. 1988, in Meteorites and the Early Solar System, ed. J. F. Kerridge \& M. S. Matthews (Tucson, AZ: Univ. Arizona Press), 819

Danger, G., Bossa, J.-B., de Marcellus, P., et al. 2011, A\&A, 525, 30

Danger, G., Fresneau, A., Abou Mrad, N., et al. 2016, GeCoA, 189, 184

Danger, G., Orthous-Daunay, F.-R., de Marcellus, P., et al. 2013, GeCoA, 118,184

d'Hendecourt, L. B., Allamandola, L. J., Baas, F., \& Greenberg, J. M. 1982, A\&A, 109, L12

d'Hendecourt, L. B., Allamandola, L. J., Grim, R. J. A., \& Greenberg, J. M. 1986, A\&A, 158, 119

d'Hendecourt, L. L. S. 2011, EPJWC, 18, 06001

Dartois, E. 2005, SSRv, 119, 293

de Marcellus, P. 2010, PhD thesis, Univ. Paris-Sud XI, France 
de Marcellus, P., Bertrand, M., Nuevo, M., et al. 2011a, AsBio, 11, 847 de Marcellus, P., Fresneau, A., Brunetto, R., et al. 2017, MNRAS, 464, 114 de Marcellus, P., Meinert, C., Myrgorodska, I., et al. 2015, PNAS, 112, 965 de Marcellus, P., Meinert, C., Nuevo, M., et al. 2011b, ApJL, 727, L27 Ehrenfreund, P., Glavin, D. P., Botta, O., et al. 2001, PNAS, 98, 2138

Elsila, J. E., Aponte, J. C., Blackmond, D. G., et al. 2016, ACS Cent. Sci., 2, 370

Elsila, J. E., Charnley, S. B., \& Burton, A. S. 2012, M\&PS, 47, 1517

Elsila, J. E., Dworkin, J. P., Bernstein, M. P., et al. 2007, ApJ, 660, 911

Fresneau, A., Abou Mrad, N., d'Hendecourt, L. L. S., et al. 2017, ApJ, 837, 168

Glavin, D. P., Callahan, M. P., Dworkin, J. P., et al. 2010, M\&PS, 45, 1948

Glavin, D. P., Dworkin, J. P., Aubrey, A., et al. 2006, M\&PS, 41, 889

Greenberg, J. M. 1978, in Cosmic Dust, ed. J. A. M. McDonnell (New York: Wiley), 187

Greenberg, J. M., \& Yencha, A. J. 1973, in IAU Symp. 52, Interstellar Dust and Related Topics, ed. J. M. Greenberg \& H. C. van de Hulst (Dordrecht: Reidel), 369

Grimm, R. E., \& McSween, H. Y. 1989, Icar, 82, 244

Hagen, W., Allamandola, L. J., \& Greenberg, J. M. 1979, Ap\&SS, 65, 215

Herd, C. D. K., Blinova, A., Simkus, D. N., et al. 2011, Sci, 332, 1304

Hewins, R. H., Bourot-Denise, M., Zanda, B., et al. 2014, GeCoA, 124, 190

Hudson, R. L., \& Moore, M. H. 2000, Icar, 145, 661

Hudson, R. L., Moore, M. H., Dworkin, J. P., Martin, M. P., \& Pozun, Z. D. 2008, AsBio, 8, 771

Kaiser, R. I. 2015, IAUGA, 29, 2231728

Kaiser, R. I., Stockton, A. M., Kim, Y. S., et al. 2014, ApJ, 765, 111

Kasamatsu, T., Kaneko, T., Saito, T., et al. 1997, Bull. Chem. Soc. Jpn., 70,1021

King, A. J., Schofield, P. F., \& Russell, S. S. 2017, M\&PS, 52, 1197

Lerner, N. R., \& Cooper, G. W. 2005, GeCoA, 69, 2901

Marrocchi, Y., Gounelle, M., Blanchard, I., et al. 2014, M\&PS, 49, 1232

Martins, Z. 2011, Elements, 7, 35

Martins, Z., Alexander, C. M. O’. D., Orzechowska, G. E., et al. 2007, M\&PS, 42,2125

Martins, Z., Cottin, H., Kotler, J. M., et al. 2017, SSRv, 209, 43

Martins, Z., Modica, P., Zanda, B., et al. 2015, M\&PS, 50, 926

Martins, Z., \& Sephton, M. A. 2009, in Amino Acids, Peptides and Proteins in Organic Chemistry: Origins and Synthesis of Amino Acids, ed. H. H. Weinheim (Wiley-VCH), 3

McSween, H. Y. 1979, RvGeo, 17, 1059
Meinert, C., Filippi, J. J., de Marcellus, P., et al. 2012, Chem. Plus Chem., 77,186

Meinert, C., Myrgorodska, I., de Marcellus, P., et al. 2016, Sci, 352, 208

Merouane, S., Djouadi, Z., d'Hendecourt, L., et al. 2012, ApJ, 756, 154

Messenger, S. 2000, Natur, 404, 968

Modica, P., Meinert, C., de Marcellus, P., et al. 2014, ApJ, 788, 79

Muñoz Caro, G. M., Dartois, E., Boduch, P., et al. 2014, A\&A, 566, 93

Muñoz Caro, G. M., Meierhenrich, U., Schutte, W. A., et al. 2004, A\&A, 413, 209

Muñoz Caro, G. M., Meierhenrich, U. J., Schutte, W. A., et al. 2002, Natur, 416, 403

Muñoz-Caro, G. M., \& Schutte, W. A. 2003, A\&A, 412, 121

Myrgorodska, I., Meinert, C., Martins, Z., et al. 2015, Angew. Chem. Int. ed., 54,1402

Nakamura-Messenger, K., Messenger, S., Keller, L. P., et al. 2006, Sci, 314, 1439

Nuevo, M., Bredehöft, J. H., Meierhenrich, U. J., et al. 2010, AsBio, 10, 245

Nuevo, M., Chen, Y.-J., Yih, T.-S., et al. 2007, AdSpR, 40, 1628

Nuevo, M., Meierhenrich, U. J., Muñoz Caro, G. M., et al. 2006, A\&A, 457, 741

Nuevo, M., Milam, S. N., Sandford, S. A., et al. 2009, AsBio, 9, 683

Nuevo, M., Sandford, S. A., \& Cooper, G. 2017, LPI, 48, 2496

Nuevo, M. L., Auger, G., Blanot, \& D'Hendecourt, L. 2008, OLEB, 38, 37

Oro, J. 1961, Natur, 190, 389

Palmer, E. E., \& Lauretta, D. S. 2011, M\&PS, 46, 1587

Peltzer, E. T., \& Bada, J. L. 1978, Natur, 272, 443

Piani, L., Yurimoto, H., Remusat, L., \& Gonzales, A. 2017, In situ Hydrogen Isotopic Composition of H-Bearing Phases in the Matrix of CM Carbonaceous Chondrites, https://goldschmidt.info/2017/abstracts/abstractView? id $=2017002891$

Rubin, A. E., Trigo-Rodríguez, J. M., Huber, H., et al. 2007, GeCoA, 71, 2361

Sephton, M. A. 2002, Nat. Prod. Rep., 19, 292

Shimoyama, A., \& Ogasawara, R. 2002, OLEB, 32, 165

Strazzulla, G. 1997, AdSpR, 19, 1077

Tomeoka, K., \& Buseck, P. R. 1985, GeCoA, 49, 2149

Vinogradoff, V., Bernard, S., Le Guillou, C., et al. 2018, Icar, 305, 358

Vinogradoff, V., Le Guillou, C., Bernard, S., et al. 2017, GeCoA, 212, 234

Yabuta, H., Williams, L. B., Cody, G. D., et al. 2007, M\&PS, 42, 37

Zolensky, M., \& McSween, H. Y. 1988, in Meteorites and the Early Solar System, ed. J. F. Kerridge \& M. S. Matthews (Tucson, AZ: Univ. Arizona Press), 114 